\title{
MSSM Dark Matter in Light of Higgs and LUX Results
}

\author{
W. Abdallah ${ }^{1,2}$ and S. Khalil ${ }^{1}$ \\ ${ }^{1}$ Center for Fundamental Physics, Zewail City of Science and Technology, 6th of October City, Giza 12588, Egypt \\ ${ }^{2}$ Department of Mathematics, Faculty of Science, Cairo University, Giza 12613, Egypt
}

Correspondence should be addressed to W. Abdallah; wabdallah@zewailcity.edu.eg

Received 26 September 2015; Accepted 6 December 2015

Academic Editor: Enrico Lunghi

Copyright (C) 2016 W. Abdallah and S. Khalil. This is an open access article distributed under the Creative Commons Attribution License, which permits unrestricted use, distribution, and reproduction in any medium, provided the original work is properly cited. The publication of this article was funded by SCOAP ${ }^{3}$.

\begin{abstract}
The constraints imposed on the Minimal Supersymmetric Standard Model (MSSM) parameter space by the Large Hadron Collider (LHC) Higgs mass limit and gluino mass lower bound are revisited. We also analyze the thermal relic abundance of lightest neutralino, which is the Lightest Supersymmetric Particle (LSP). We show that the combined LHC and relic abundance constraints rule out most of the MSSM parameter space except a very narrow region with very large $\tan \beta(\sim 50)$. Within this region, we emphasize that the spin-independent scattering cross section of the LSP with a proton is less than the latest Large Underground Xenon (LUX) limit by at least two orders of magnitude. Finally, we argue that nonthermal Dark Matter (DM) scenario may relax the constraints imposed on the MSSM parameter space. Namely, the following regions are obtained: $m_{0} \simeq \mathcal{O}(4) \mathrm{TeV}$ and $m_{1 / 2} \simeq 600 \mathrm{GeV}$ for low $\tan \beta(\sim 10) ; m_{0} \sim m_{1 / 2} \simeq \mathcal{O}(1) \mathrm{TeV}$ or $m_{0} \simeq \mathcal{O}(4) \mathrm{TeV}$ and $m_{1 / 2} \simeq 700 \mathrm{GeV}$ for large $\tan \beta(\sim 50)$.
\end{abstract}

\section{Introduction}

The most recent observations by the Planck satellite confirmed that $26.8 \%$ of the universe content is in the form of DM and the usual visible matter only accounts for $5 \%$ [1]. The LSP remains one of the best candidates for the DM $[2,3]$. It is a Weakly Interacting Massive Particle (WIMP) that can naturally account for the observed relic density of DM.

Despite the absence of direct experimental verification, Supersymmetry (SUSY) is still the most promising candidate for a unified theory beyond the Standard Model (SM). SUSY is a generalization of the space-time symmetries of the quantum field theory that links the matter particles (quarks and leptons) with the force-carrying particles and implies that there are additional "superparticles" necessary to complete the symmetry. In this regard, SUSY solves the problem of the quadratic divergence in the Higgs sector of the SM in a very elegant natural way. The most simple supersymmetric extension of the SM, which is the most widely studied, is known as the MSSM [4-11]. In this model, certain universality of soft SUSY breaking terms is assumed at grand unification scale. Therefore, the SUSY spectrum is determined by the following four parameters: universal scalar mass $m_{0}$, universal gaugino mass $m_{1 / 2}$, universal trilinear coupling $A_{0}$, and the ratio of the vacuum expectation values of Higgs bosons $\tan \beta$. In addition, due to $R$-parity conservation, SUSY particles are produced or destroyed only in pairs and therefore the LSP is absolutely stable, implying that it might constitute a possible candidate for DM, as first suggested by Goldberg in 1983 [12]. So although the original motivation of SUSY has nothing to do with the DM problem, it turns out that it provides a stable neutral particle and, hence, a candidate for solving the DM problem.

The landmark discovery of the SM-like Higgs boson at the LHC, with mass $\sim 125 \mathrm{GeV}[13,14]$, might be an indication for the presence of SUSY. Indeed, the MSSM predicts that there is an upper bound of $130 \mathrm{GeV}$ on the Higgs mass. However, this mass of lightest Higgs boson implies that the SUSY particles are quite heavy. This may justify the negative searches for SUSY at the LHC run-I [15-18]. However, it is clearly generating a new "little hierarchy problem."

Moreover, the relic density data [1] and upper limits on the DM scattering cross sections on nuclei (LUX [19] and other direct detection experiments $[20,21]$ ) impose stringent constraints on the parameter space of the MSSM [22-25]. In fact, combining the collider, astrophysics, and rare decay 
constraints [26-36] almost rules out the MSSM. It is tempting therefore to explore well motivated extensions of the MSSM, such as NMSSM [37, 38] and BLSSM [39, 40], which may alleviate the little hierarchy problem of the MSSM through additional contributions to Higgs mass [37, 38, 41] and also provide new DM candidates [42-45] that may account for the relic density with no conflict with other phenomenological constraints.

In this paper, we analyze the constraints imposed by the Higgs mass limit and the gluino lower bound, which are the most stringent collider constraints, on the constrained MSSM (minimal SUGRA model, hereafter referred to as MSSM) parameter space. In particular, these constraints imply that the gaugino mass, $m_{1 / 2}$, resides within the mass range: $620 \mathrm{GeV} \lesssim m_{1 / 2} \lesssim 2000 \mathrm{GeV}$, while the other parameters are much less constrained. We study the effect of the measured DM relic density on the MSSM allowed parameter space. We emphasized that in this case all parameter space is ruled out except for few points around $\tan \beta \sim 50, m_{0} \sim 1 \mathrm{TeV}$, and $m_{1 / 2} \sim 1.5 \mathrm{TeV}$. We also investigate the direct detection rate of the LSP at these allowed points in light of the latest LUX result. Finally, we show that if one assumes nonstandard scenario of cosmology with low reheating temperature, where the LSP may reach equilibrium before the reheating time, then the relic abundance constraints on $\left(m_{0}, m_{1 / 2}\right)$ can be significantly relaxed.

The paper is organized as follows. In Section 2, we briefly introduce the MSSM and study the constraints on $\left(m_{0}, m_{1 / 2}\right)$ plane from Higgs and gluino mass experimental limits. In Section 3, we study the thermal relic abundance of the LSP in the allowed region of parameter space. We show that the combined LHC and relic abundance constraints rule out most of the parameter space except the case of very large $\tan \beta$. We also provide the expected rate of direct LSP detection at these points with large $\tan \beta$ and $\mathrm{TeV}$ masses. Section 4 is devoted to nonthermal scenario of DM and how it can relax the constraints imposed on MSSM parameter space. Finally, we give our conclusions in Section 5.

\section{MSSM after the LHC Run-I}

The particle content of the MSSM is three generations of (chiral) quark and lepton superfields; the (vector) superfields are necessary to gauge $S U(3)_{C} \times S U(2)_{L} \times U(1)_{Y}$ gauge of the SM, and two (chiral) SU(2) doublet Higgs superfields. The introduction of a second Higgs doublet is necessary in order to cancel the anomalies produced by the fermionic members of the first Higgs superfield and also to give masses to both up and down type quarks. The interactions between Higgs and matter superfields are described by the superpotential

$$
\begin{aligned}
W= & h_{U} Q_{L} U_{L}^{c} H_{2}+h_{D} Q_{L} D_{L}^{c} H_{1}+h_{L} L_{L} E_{L}^{c} H_{1} \\
& +\mu H_{1} H_{2} .
\end{aligned}
$$

Here, $Q_{L}$ contains $S U(2)$ (s) quark doublets and $U_{L}^{c}, D_{L}^{c}$ are the corresponding singlets, (s)lepton doublets and singlets reside in $L_{L}$ and $E_{L}^{c}$, respectively. $H_{1}$ and $H_{2}$ denote Higgs superfields with hypercharge $Y=\mp 1 / 2$. Further, due to the fact that Higgs and lepton doublet superfields have the same
$S U(3)_{C} \times S U(2)_{L} \times U(1)_{Y}$ quantum numbers, we have additional terms that can be written as

$$
\begin{aligned}
W^{\prime}= & \lambda_{i j k} L_{i} L_{j} E_{k}^{c}+\lambda_{i j k}^{\prime} L_{i} Q_{j} D_{k}^{c}+\lambda_{i j k}^{\prime \prime} D_{i}^{c} D_{j}^{c} U_{k}^{c} \\
& +\mu_{i} L_{i} H_{2} .
\end{aligned}
$$

These terms violate baryon and lepton number explicitly and lead to proton decay at unacceptable rates. To forbid these terms, a new symmetry, called $R$-parity, is introduced, which is defined as $R_{P}=(-1)^{3 B+L+2 S}$, where $B$ and $L$ are baryon and lepton number and $S$ is the spin. There are two remarkable phenomenological implications of the presence of $R$-parity: (i) SUSY particles are produced or destroyed only in pair; (ii) the LSP is absolutely stable and, hence, it might constitute a possible candidate for DM.

In the MSSM, a certain universality of soft SUSY breaking terms at grand unification scale $M_{X}=3 \times 10^{16} \mathrm{GeV}$ is assumed. These terms are defined as $m_{0}$, the universal scalar soft mass, $m_{1 / 2}$, the universal gaugino mass, $A_{0}$, the universal trilinear coupling, $B$, and the bilinear coupling (the soft mixing between the Higgs scalars). In order to discuss the physical implication of soft SUSY breaking at low energy, we need to renormalize these parameters from $M_{X}$ down to electroweak scale, which has been performed using SARAH [46], and the spectrum has been calculated using SPheno [47, 48]. In addition, the MSSM contains another two free SUSY parameters: $\mu$ and $\tan \beta=\left\langle H_{2}\right\rangle /\left\langle H_{1}\right\rangle$. Two of these free parameters, $\mu$ and $B$, can be determined by the electroweak breaking conditions:

$$
\begin{aligned}
\mu^{2} & =\frac{m_{H_{1}}^{2}-m_{H_{2}}^{2} \tan ^{2} \beta}{\tan ^{2} \beta-1}-\frac{M_{Z}^{2}}{2}, \\
\sin 2 \beta & =\frac{-2 m_{3}^{2}}{m_{1}^{2}+m_{2}^{2}} .
\end{aligned}
$$

Thus, the MSSM has only four independent free parameters, $m_{0}, m_{1 / 2}, A_{0}, \tan \beta$, besides the sign of $\mu$, which determine the whole spectrum.

In the MSSM, the mass of the lightest Higgs state can be approximated, at the one-loop level, as [49-52]

$$
m_{h}^{2} \leq M_{Z}^{2}+\frac{3 g^{2}}{16 \pi^{2} M_{W}^{2}} \frac{m_{t}^{4}}{\sin ^{2} \beta} \log \left(\frac{m_{\tilde{t}_{1}}^{2} m_{\tilde{t}_{2}}^{2}}{m_{t}^{4}}\right) .
$$

Therefore, if one assumes that the stop masses are of order $\mathrm{TeV}$, then the one-loop effect leads to a correction of order $\mathcal{O}(100) \mathrm{GeV}$, which implies that

$$
m_{h}^{\mathrm{MSSM}} \lesssim \sqrt{(90 \mathrm{GeV})^{2}+(100 \mathrm{GeV})^{2}} \simeq 135 \mathrm{GeV} .
$$

The two-loop corrections reduce this upper bound by few GeVs [53-55]. Hence, the MSSM predicts the following upper bound for the Higgs mass: $m_{h} \lesssim 130 \mathrm{GeV}$, which was consistent with the measured value of Higgs mass (of order $125 \mathrm{GeV}$ ) at the LHC $[13,14]$.

In Figure 1, we display the contour plot of the SMlike Higgs boson: $m_{h} \in[124,126] \mathrm{GeV}$ in $\left(m_{0}, m_{1 / 2}\right)$ 

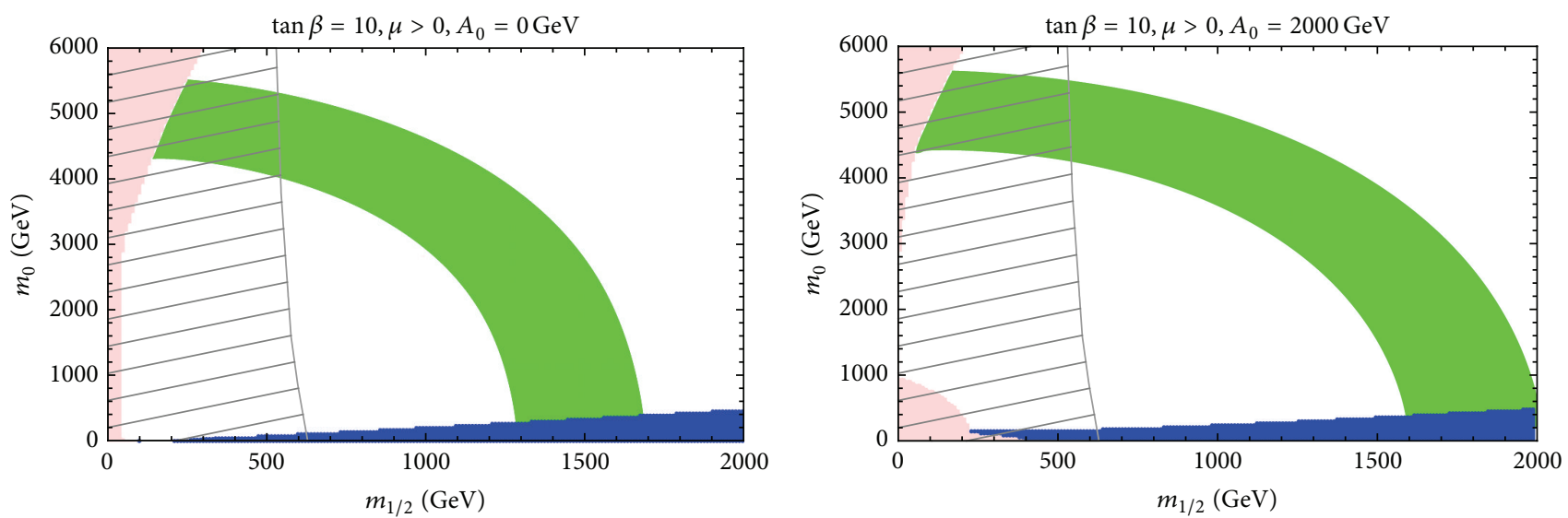

(a)
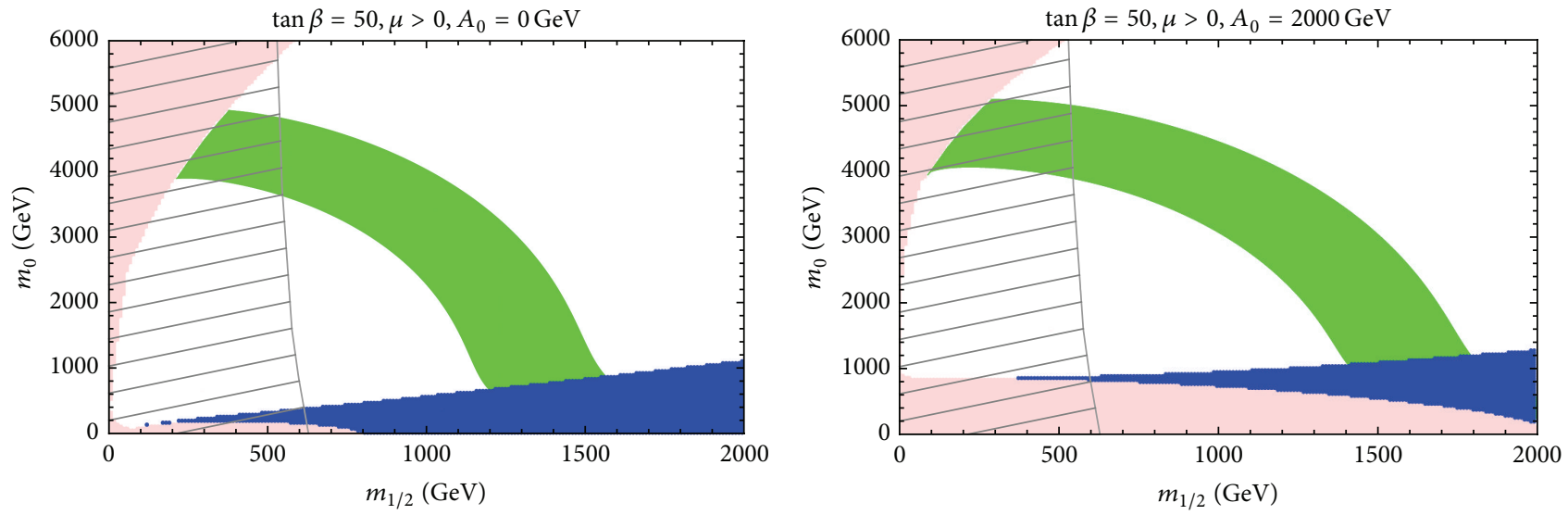

(b)

Figure 1: MSSM parameter space for $\tan \beta=10$ (a) and 50 (b) with $A_{0}=0$ and $2 \mathrm{TeV}$. The green region indicates $124 \lesssim m_{h} \lesssim 126 \mathrm{GeV}$. The blue region is excluded because the lightest neutralino is not the LSP. The pink region is excluded due to the absence of radiative electroweak symmetry breaking ( $\mu^{2}$ becomes negative). The gray shadow lines denote the excluded area because of $m_{\tilde{g}}<1.4 \mathrm{TeV}$.

plane for different values of $A_{0}$ and $\tan \beta$. It is remarkable that the smaller the value of $A_{0}$ is, the smaller the value of $m_{1 / 2}$ is needed to satisfy this value of Higgs mass. It is also clear that the scalar mass $m_{0}$ remains essentially unconstrained by Higgs mass limit. It can vary from few hundred GeVs to few TeVs. Such large values of $m_{1 / 2}$ seem to imply a quite heavy SUSY spectrum, much heavier than the lower bound imposed by direct searches at the LHC experiments in centre of mass energies $\sqrt{s}=7,8 \mathrm{TeV}$ and total integrated luminosity of order $20 \mathrm{fb}^{-1}$. Furthermore, the LHC lower limit on the gluino mass, $m_{\tilde{g}} \gtrsim 1.4 \mathrm{TeV}$ [56, 57], excluded the values of $m_{1 / 2}<620 \mathrm{GeV}$ which was allowed by Higgs mass constraints for $m_{0}>4 \mathrm{TeV}$.

$$
M_{N}=\left(\begin{array}{cc}
M_{1} & 0 \\
0 & M_{2} \\
-M_{Z} \cos \beta \sin \theta_{W} & M_{Z} \cos \beta \cos \theta_{W} \\
M_{Z} \sin \beta \sin \theta_{W} & -M_{Z} \sin \beta \cos \theta_{W}
\end{array}\right.
$$

Furthermore, this region is shown with dashed lines in Figure 1.

\section{Dark Matter Constraints on MSSM Parameter Space}

3.1. The LSP as Dark Matter Candidate. The neutralinos $\chi_{i}$ $(i=1,2,3,4)$ are the physical (mass) superpositions of two fermionic partners of the two neutral gauge bosons, called gaugino $\widetilde{B}^{0}$ (bino) and $\widetilde{W}_{3}^{0}$ (wino), and of the two neutral Higgs bosons, called Higgsinos $\widetilde{H}_{1}^{0}$ and $\widetilde{H}_{2}^{0}$. The neutralino mass matrix is given by [58-61]

$$
\left.\begin{array}{cc}
-M_{Z} \cos \beta \sin \theta_{W} & M_{Z} \sin \beta \sin \theta_{W} \\
M_{Z} \cos \beta \cos \theta_{W} & -M_{Z} \sin \beta \cos \theta_{W} \\
0 & -\mu \\
-\mu & 0
\end{array}\right),
$$


where $M_{1}$ and $M_{2}$ are related due to the universality of the gaugino masses at the grand unification scale, $M_{1}=$ $\left(3 g_{1}^{2} / 5 g_{2}^{2}\right) M_{2}$, where $g_{1}, g_{2}$ are the gauge couplings of $U(1)_{Y}$ and $S U(2)_{L}$, respectively. This Hermitian matrix is diagonalized by a unitary transformation of the neutralino fields, $M_{N}^{\text {diag }}=N^{\dagger} M_{N} N$. The lightest eigenvalue of this matrix and the corresponding eigenstate, say $\chi$, has good chance of being the LSP. The lightest neutralino will be a linear combination of the original fields:

$$
\chi=N_{11} \widetilde{B}^{0}+N_{12} \widetilde{W}^{0}+N_{13} \widetilde{H}_{1}^{0}+N_{14} \widetilde{H}_{2}^{0} .
$$

The phenomenology and cosmology of the neutralino are governed primarily by its mass and composition. A useful parameter for describing the neutralino composition is the gaugino "purity" function $f_{g}=\left|N_{11}\right|^{2}+\left|N_{12}\right|^{2}$ [58-61]. If $f_{g}>$ 0.5 , then the neutralino is primarily gaugino and if $f_{g}<0.5$, then the neutralino is primarily Higgsino. Actually, if $|\mu|>$ $\left|M_{2}\right| \geq M_{Z}$, the two lightest neutralino states will be determined by the gaugino components; similarly, the light chargino will be mostly a charged wino, while if $|\mu|<\left|M_{2}\right|$, the two lighter neutralinos and the lighter chargino are all mostly Higgsinos, with mass close to $|\mu|$. Finally, if $|\mu| \simeq\left|M_{2}\right|$, the states will be strongly mixed.

Here, two remarks are in order. (i) The abovementioned constraints in $m_{1 / 2}$ from Higgs mass limit and gluino mass lower bound imply that $m_{\chi} \gtrsim 240 \mathrm{GeV}$, which is larger than the limits obtained from direct searches at the LHC. Moreover, an upper bound of order one $\mathrm{TeV}$ is also obtained (from Higgs mass constraint). (ii) In this region of allowed parameter space, the LSP is essentially pure bino, as shown in Figure 2 . This can be easily understood from the fact that $\mu$ parameter, determined by the radiative electroweak breaking condition, (3), is typically of order $m_{0}$ and hence it is much heavier than the gaugino mass $M_{1}$.

3.2. Relic Density. As advocated in the previous section, the LSP in MSSM, the lightest neutralino $\chi$, is a perfect candidate for DM. Here, we assume that $\chi$ was in thermal equilibrium with the SM particles in the early universe and decoupled when it was nonrelativistic. Once $\chi$ annihilation rate $\Gamma_{\chi}=$ $\left\langle\sigma_{\chi}^{\text {ann }} v\right\rangle n_{\chi}$ dropped below the expansion rate of the universe, $\Gamma_{\chi} \leq H$, the LSP particles stop to annihilate and fall out of equilibrium and their relic density remains intact till now. The above $\left\langle\sigma_{\chi}^{\text {ann }} v\right\rangle$ refers to thermally averaged total cross section for annihilation of $\chi \chi$ into lighter particles times the relative velocity, $v$.

The relic density is then determined by the Boltzmann equation for the LSP number density $\left(n_{\chi}\right)$ and the law of entropy conservation:

$$
\begin{aligned}
& \frac{d n_{\chi}}{d t}=-3 H n_{\chi}-\left\langle\sigma_{\chi}^{\mathrm{ann}} v\right\rangle\left[\left(n_{\chi}\right)^{2}-\left(n_{\chi}^{\mathrm{eq}}\right)^{2}\right], \\
& \frac{d s}{d t}=-3 H s,
\end{aligned}
$$

where $n_{\chi}^{\mathrm{eq}}$ is the LSP equilibrium number density which, as a function of temperature $T$, is given by $n_{\chi}^{\mathrm{eq}}=$

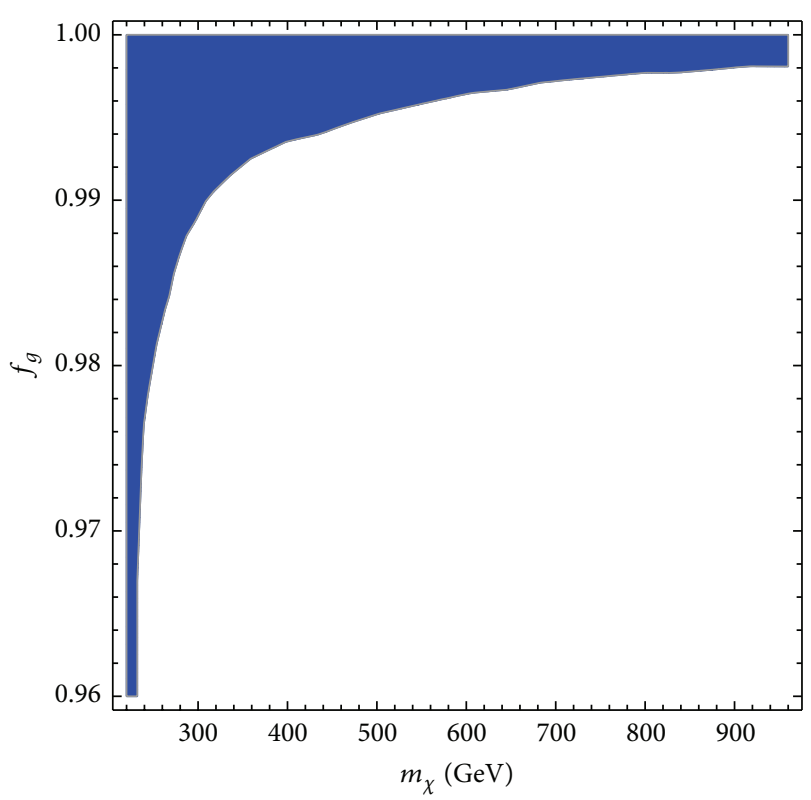

FIGURE 2: The mass of lightest neutralino versus the purity function in the region of parameter space allowed by gluino and Higgs mass limits.

$g_{\chi}\left(m_{\chi} T / 2 \pi\right)^{3 / 2} e^{-m_{\chi} / T}$. Here, $m_{\chi}$ and $g_{\chi}$ are the mass and the number of degrees of freedom of the LSP, respectively. Finally, $s$ is the entropy density. In the standard cosmology, the Hubble parameter $H$ is given by $H(T)=2 \pi \sqrt{\pi g_{*} / 45}\left(T^{2} / M_{P l}\right)$, where $M_{P l}=1.22 \times 10^{19} \mathrm{GeV}$ and $g_{*}$ is the number of relativistic degrees of freedom, for MSSM $g_{*} \simeq 228.75$. Let us introduce the variable $x=m_{\chi} / T$ and define $Y=n_{\chi} / s$ with $Y_{\mathrm{eq}}=n_{\chi}^{\mathrm{eq}} / s$. In this case, the Boltzmann equation is given by

$$
\frac{d Y}{d x}=\frac{1}{3 H} \frac{d s}{d x}\left\langle\sigma_{\chi}^{\mathrm{ann}} v\right\rangle\left(Y^{2}-Y_{\mathrm{eq}}^{2}\right) .
$$

In radiation domination era, the entropy, as a function of the temperature, is given by

$$
s(x)=\frac{2 \pi^{2}}{45} g_{*_{s}}(x) m_{\chi}^{3} x^{-3},
$$

which is deduced from the fact that $s=(\rho+p) / T$ and $g_{*}$ is the effective degrees of freedom for the entropy density. Therefore, one finds

$$
\frac{d s}{d x}=-\frac{3 s}{x} .
$$

Thus, with assuming $g_{*} \simeq g_{*_{s}}$, the following expression for the Boltzmann equation for the LSP number density is obtained:

$$
\frac{d Y}{d x}=-\sqrt{\frac{\pi g_{*}}{45}} M_{P l} m_{\chi} \frac{\left\langle\sigma_{\chi}^{\mathrm{ann}} v\right\rangle}{x^{2}}\left(Y^{2}-Y_{\mathrm{eq}}^{2}\right) .
$$

If one considers the s-wave and p-wave annihilation processes only, the thermal average $\left\langle\sigma_{\chi}^{\text {ann }} v\right\rangle$ then shows as

$$
\left\langle\sigma_{\chi}^{\mathrm{ann}} v\right\rangle \simeq a_{\chi}+\frac{6 b_{\chi}}{x},
$$




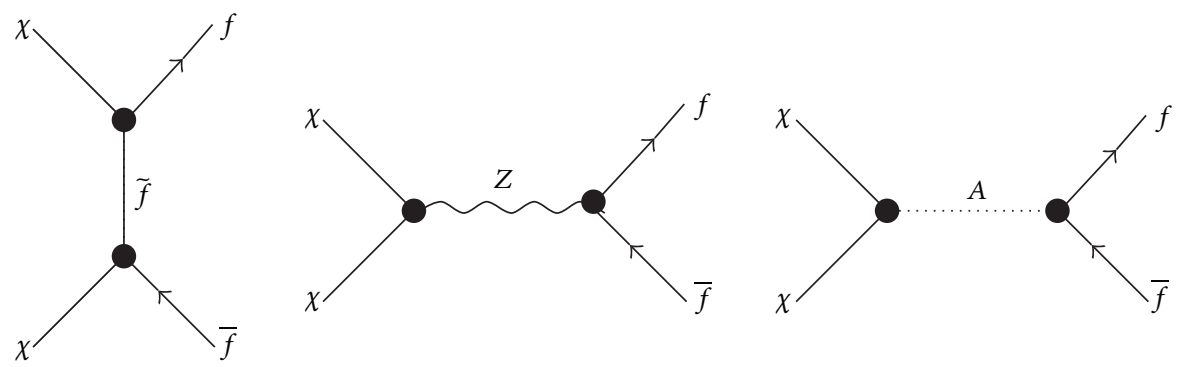

FIgURE 3: Feynman diagrams contributing to early-universe neutralino $\chi$ annihilation into fermions through sfermions, $Z$-gauge boson, and Higgs.

where $a_{\chi}$ and $b_{\chi}$ are the s-wave and p-wave contributions of annihilation processes, respectively. The relic density of the $\mathrm{DM}$ candidate is given by

$$
\Omega h^{2}=\frac{m_{\chi} s_{0} Y_{\chi}(\infty)}{\rho_{c} / h^{2}},
$$

where $s_{0}=2282.15 \times 10^{-41} \mathrm{GeV}^{3}, \rho_{c}=8.0992 h^{2} \times 10^{-47} \mathrm{GeV}^{4}$, and by solving the Boltzmann equation, one can find $Y_{\chi}(\infty)$ as follows [62]:

$$
Y_{\chi}(\infty)=\frac{1}{\lambda_{\chi}}\left(\frac{a_{\chi}}{x\left(T_{f}\right)}+\frac{3 b_{\chi}}{x^{2}\left(T_{f}\right)}\right)^{-1},
$$

where $T_{f}$ is the freeze-out temperature, $\lambda_{\chi}=s\left(m_{\chi}\right) / H\left(m_{\chi}\right)$, and $x\left(T_{f}\right)$ is given by

$$
x\left(T_{f}\right)=\ln \left[\frac{\alpha_{\chi} \lambda_{\chi} c(c+2)}{\sqrt{x\left(T_{f}\right)}}\left(a_{\chi}+\frac{6 b_{\chi}}{x\left(T_{f}\right)}\right)\right],
$$

where $\alpha_{\chi}=\left(45 / 2 \pi^{4}\right) \sqrt{\pi / 8}\left(g_{\chi} / g_{*_{s}}\left(T_{f}\right)\right)$; the value $c=1 / 2$ results in a typical accuracy of about $5-10 \%$ more than sufficient for our purposes here.

The lightest neutralino may annihilate into fermionantifermion $(f \bar{f}), W^{+} W^{-}, Z Z, W^{+} H^{-}, Z A, Z H, Z h$, and $H^{+} H^{-}$and all other contributions of neutral Higgs. For a bino-like LSP, that is, $N_{11} \simeq 1$ and $N_{1 i} \simeq 0, i=2,3,4$, one finds that the relevant annihilation channels are the fermionantifermion ones, as shown in Figure 3, and all other channels are instead suppressed. Also, the annihilation process mediated by $Z$-gauge boson is suppressed due to the small $Z \chi \chi$ coupling $\propto N_{13}^{2}-N_{14}^{2}$, except at the resonance when $m_{\chi} \sim M_{Z} / 2$, which is no longer possible due to the abovementioned constraints. Furthermore, one finds that the tchannel annihilation (first Feynman diagram in Figure 3) is predominantly into leptons through the exchanges of the three slepton families $\left(\widetilde{l}_{L}, \widetilde{l}_{R}\right)$, with $l=e, \mu, \tau$. The squarks exchanges are suppressed due to their large masses.

In Figure 4, we display the constraint from the observed limits of $\Omega h^{2}$ on the plane $\left(m_{0}-m_{1 / 2}\right)$ for $A_{0}=0,2000 \mathrm{GeV}$, $\tan \beta=10,50$, and $\mu>0$. Here, we used micrOMEGAs [63] to compute the complete relic abundance of the lightest neutralino, taking into account the possibility of having coannihilation with the next-to-lightest supersymmetric particle, which is typically the lightest stau. Note that this type of coannihilation is not included in the approximated expressions in (14)-(17). In this figure, the red regions correspond to a relic abundance within the measured limits [1]:

$$
0.09<\Omega h^{2}<0.14
$$

It is noticeable that, with low $\tan \beta(\sim 10)$, this region corresponds to light $m_{1 / 2}(<500 \mathrm{GeV})$, where significant coannihilation between the LSP and stau took place. However, this possibility is now excluded by the Higgs and gluino mass constraints [64]. At large $\tan \beta$, another region is allowed due to possible resonance due to $s$-channel annihilation of the DM pair into fermion-antifermion via the pseudoscalar Higgs boson $A$ at $M_{A} \simeq 2 m_{\chi}$ [65]. For $A_{0}=0$, a very small part of this region is allowed by the Higgs mass constraint, while for large $A_{0}(\sim 2 \mathrm{TeV})$ slight enhancement of this part can be achieved. In Figure 5, we zoom in on this region to show the explicit dependence of the relic abundance on the LSP mass and large values of $\tan \beta$. As can be seen from this figure, there is no point that can satisfy the relic abundance stringent constraints with $\tan \beta<30$.

3.3. Direct Detection. Perhaps the most natural way of searching for the neutralino DM is provided by direct experiments, where the effects induced in appropriate detectors by neutralino-nucleus elastic scattering may be measured. The elastic-scattering cross section of the LSP with a given nucleus has two contributions: spin-dependent contribution arising from $Z$ and $\widetilde{q}$ exchange diagrams and spin-independent (scalar) contribution due to the Higgs and squark exchange diagrams, which is typically suppressed. The effective scalar interaction of neutralino with a quark is given by

$$
\mathscr{L}_{\text {scalar }}=f_{q} \bar{\chi} \chi \bar{q} q,
$$

where $f_{q}$ is the neutralino-quark effective coupling. The scalar cross section of the neutralino scattering with target nucleus at zero momentum transfer is given by [2]

$$
\sigma_{0}^{\mathrm{SI}}=\frac{4 m_{r}^{2}}{\pi}\left(Z f_{p}+(A-Z) f_{n}\right)^{2},
$$

where $Z$ and $A-Z$ are the number of protons and neutrons, respectively, $m_{r}=m_{N} m_{\chi} /\left(m_{N}+m_{\chi}\right)$, where $m_{N}$ is 

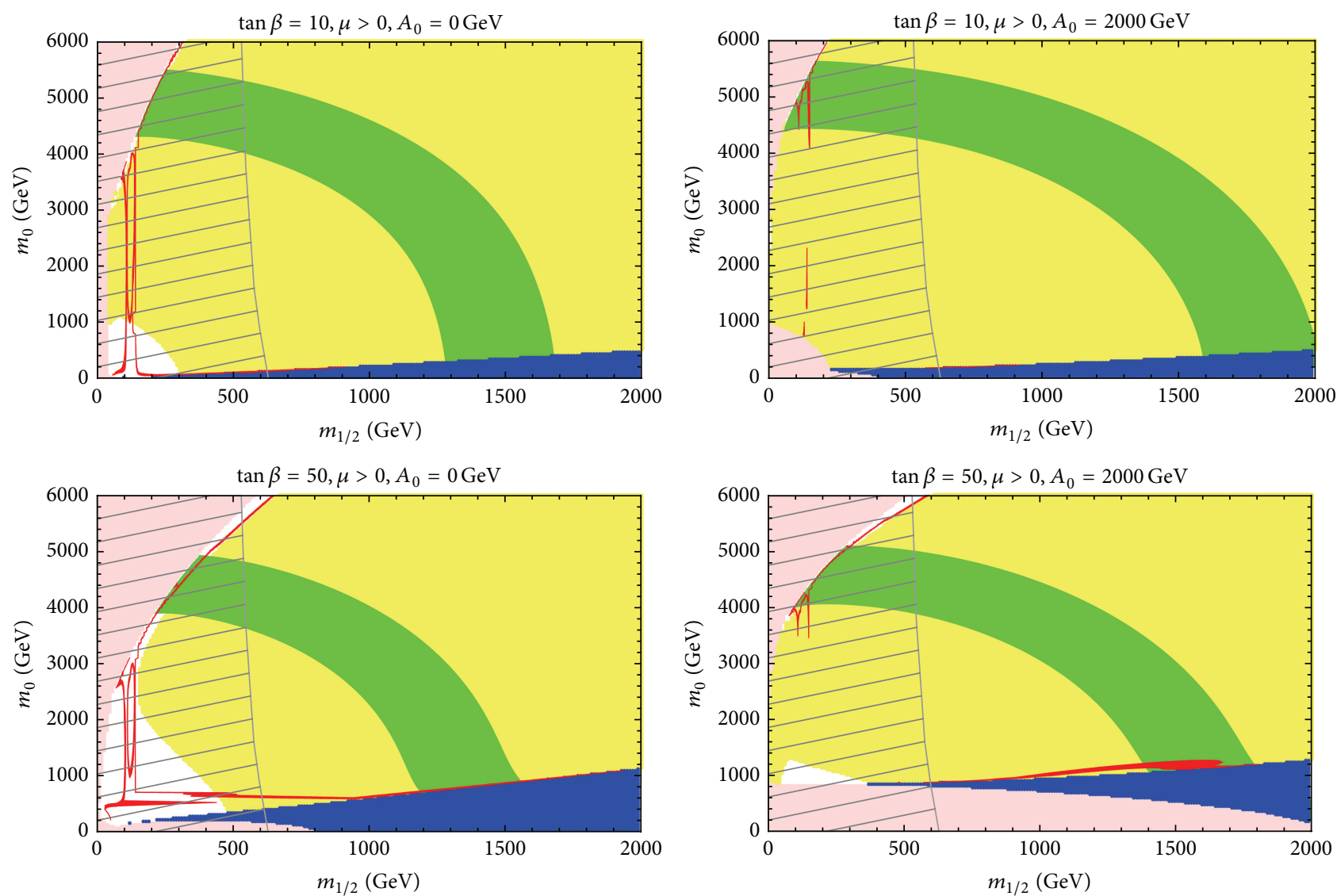

FIGURE 4: LSP relic abundance constraints (red regions) on $\left(m_{0}-m_{1 / 2}\right)$ plane for $\tan \beta$ and $A_{0}$ as in Figure 1. The LUX result is satisfied by the yellow region. The other color codes are as in Figure 1.

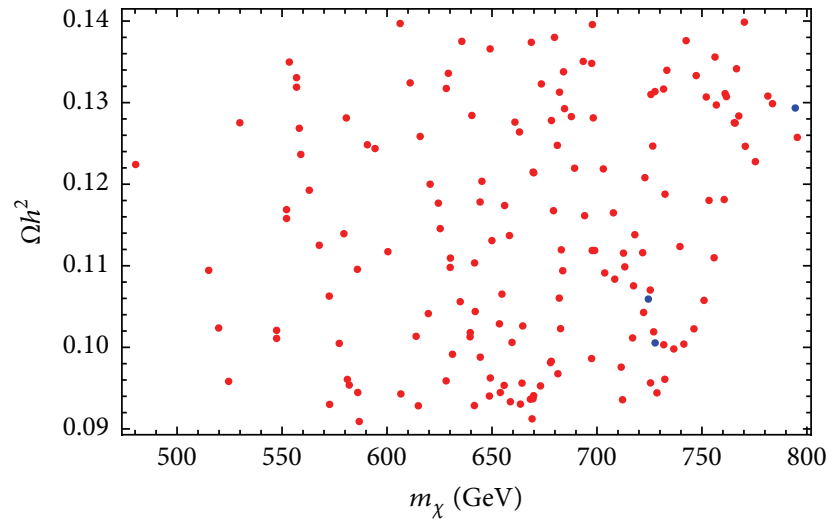

FIGURE 5: The relic abundance versus the mass of the LSP for different values of $\tan \beta$. Red points indicate $40 \leq \tan \beta \leq 50$ and blue points $30 \leq \tan \beta<40$. All points satisfy the abovementioned constraints.

the nucleus mass, and $f_{p}, f_{n}$ are the neutralino coupling to protons and neutrons, respectively. The differential scalar cross section for nonzero momentum transfer $q$ can now be written:

$$
\frac{d \sigma_{\mathrm{SI}}}{d q^{2}}=\frac{\sigma_{0}^{\mathrm{SI}}}{4 m_{r}^{2} v^{2}} F^{2}\left(q^{2}\right), \quad 0<q^{2}<4 m_{r}^{2} v^{2}
$$

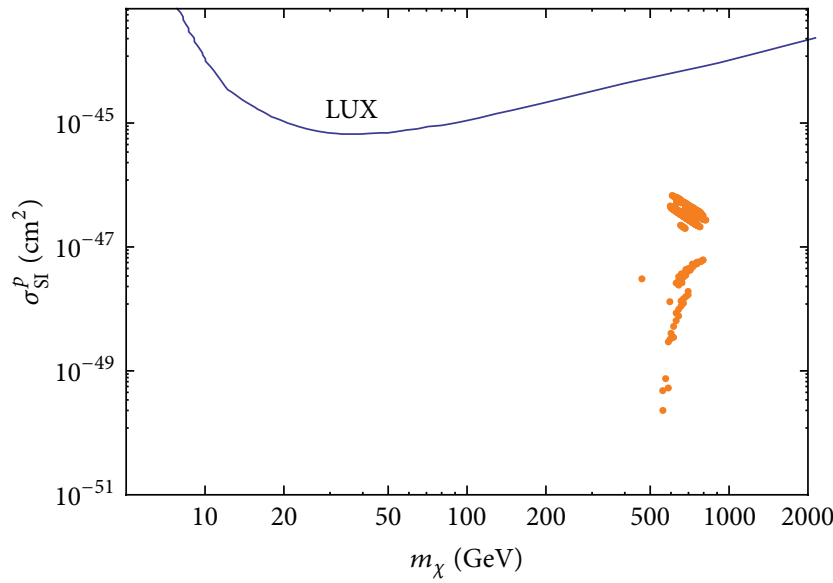

FIGURE 6: Spin-independent scattering cross section of the LSP with a proton versus the mass of the LSP within the region allowed by all constraints (from the LHC and relic abundance).

where $v$ is the neutrino velocity and $F\left(q^{2}\right)$ is the form factor [2]. In Figure 6, we display the MSSM prediction for spinindependent scattering cross section of the LSP with a proton $\left(\sigma_{\mathrm{SI}}^{p}=\left.\int_{0}^{4 m_{r}^{2} v^{2}}\left(d \sigma_{\mathrm{SI}} / d q^{2}\right)\right|_{f_{n}=f_{p}} d q^{2}\right)$ after imposing the LHC 

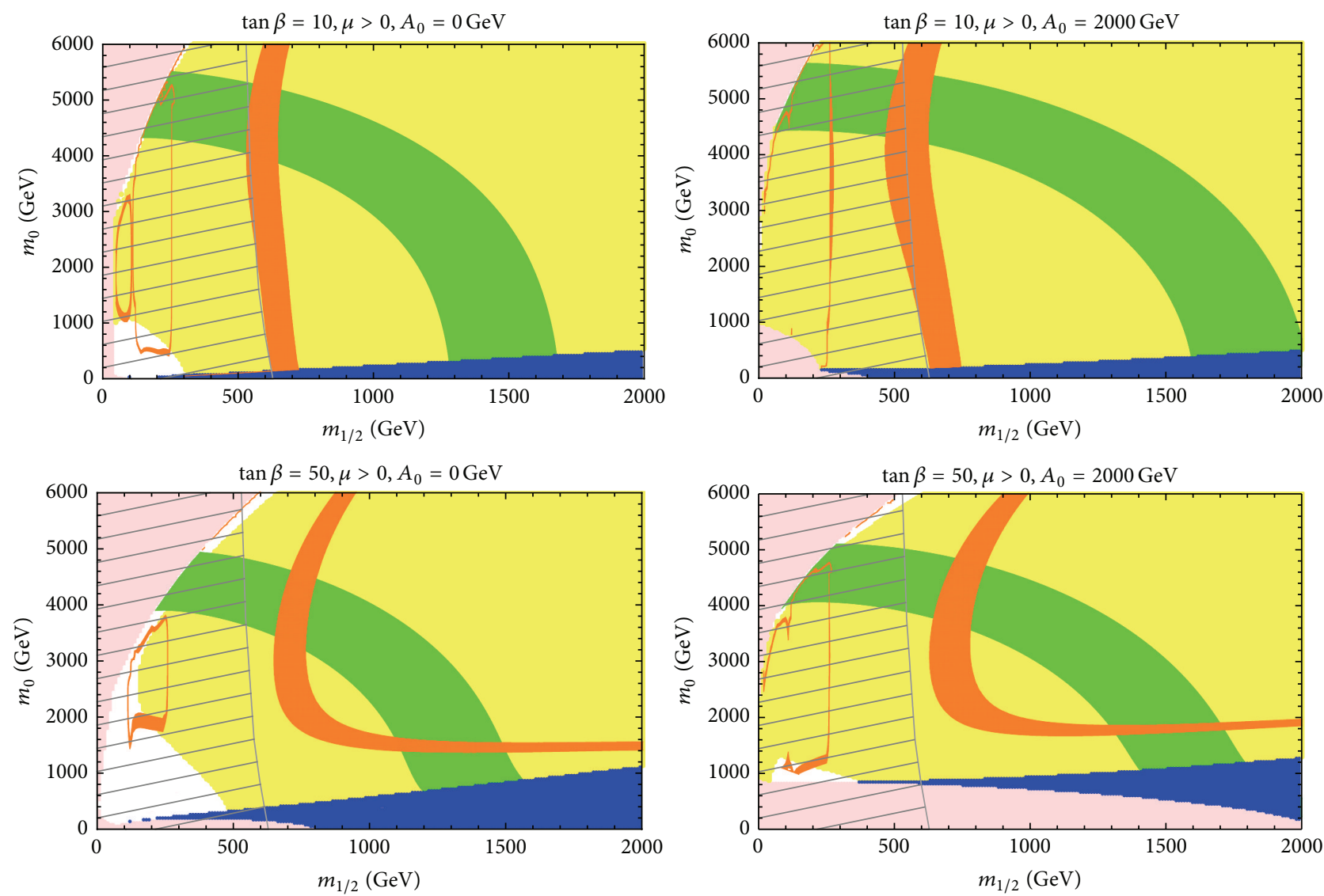

FIgURE 7: LSP nonthermal relic abundance constraints (red regions) on $\left(m_{0}-m_{1 / 2}\right)$ plane for $\tan \beta$ and $A_{0}$ as in Figure 1. The color codes are as in Figure 1.

and relic abundance constraints. It is clear that our results for $\sigma_{\mathrm{SI}}^{p}$ are less than the recent LUX bound (blue curve) by at least two orders of magnitude. This would explain the negative results of direct searches so far.

\section{Nonthermal Dark Matter and MSSM Parameter Space}

In the previous section, we assumed standard cosmology scenario where the reheating temperature $T_{\mathrm{RH}}$ is very large; namely, $T_{\mathrm{RH}} \gg T_{f} \simeq 10 \mathrm{GeV}$. However, the only constraint on the reheating temperature, which could be associated with decay of any scalar field, $\phi$, not only the inflaton field, is $T_{\mathrm{RH}} \gtrsim 1 \mathrm{MeV}$ in order not to spoil the successful predictions of big bang nucleosynthesis.

A detailed analysis of the relic density with a low reheating temperature has been carried out in [66]. It was emphasized that, for a large annihilation cross section, $\left\langle\sigma^{\text {ann }} v\right\rangle \gtrsim$ $10^{-14} \mathrm{GeV}^{-2}$ so that the neutralino reaches equilibrium before reheating, and if there are a large number of neutralinos produced by the scalar field $\phi$ decay, then the relic density is estimated as [67]

$$
\Omega h^{2}=\frac{3 m_{\chi} \Gamma_{\phi}}{2\left(2 \pi^{2} / 45\right) g_{*}\left(T_{\mathrm{RH}}\right) T_{\mathrm{RH}}^{3}\left\langle\sigma_{\chi}^{\mathrm{ann}} v\right\rangle} \frac{h^{2}}{\rho_{c} / s_{0}} .
$$

Here, the reheating temperature is defined as [62]

$$
T_{\mathrm{RH}}=\left(\frac{90}{\pi^{2} g_{*}\left(T_{\mathrm{RH}}\right)}\right)^{1 / 4}\left(\Gamma_{\phi} M_{P l}\right)^{1 / 2},
$$

where the decay width $\Gamma_{\phi}$ is given by

$$
\Gamma_{\phi}=\frac{1}{2 \pi} \frac{m_{\phi}^{3}}{\Lambda^{2}} .
$$

The scale $\Lambda$ is the effective suppression scale, which is of order the grand unification scale $M_{X}$. Therefore, for scalar field with mass $m_{\phi} \simeq 10^{7} \mathrm{GeV}$, one finds $\Gamma_{\phi} \simeq 10^{-11} \mathrm{GeV}$, and in our calculations, we have used $g_{*}=10.75$ due to the consideration of a low reheating temperature scenario.

In Figure 7, we show the constraints imposed on the $\operatorname{MSSM}\left(m_{0}-m_{1 / 2}\right)$ plane in case of nonthermal relic abundance of the LSP for $\tan \beta=10,50$ and $A_{0}=0,2 \mathrm{TeV}$. In this plot, we also imposed the LHC constraints, namely, the Higgs mass limit and the gluino mass lower bound, similar to the case of thermal scenario. It is clear from this figure that the stringent constraints imposed on the MSSM parameter space by thermal relic abundance are now relaxed and now low $\tan \beta(\sim 10)$ is allowed but with very heavy $m_{0}(\sim \mathcal{O}(4) \mathrm{TeV})$ and $m_{1 / 2} \simeq 600 \mathrm{GeV}$. In addition, the following two regions are now allowed with large $\tan \beta(\sim 50)$ : (i) $m_{0} \sim m_{1 / 2} \simeq$ 
$\mathcal{O}(1) \mathrm{TeV}$; (ii) $m_{0} \simeq \mathcal{O}$ (4) $\mathrm{TeV}$ and $m_{1 / 2} \simeq 700 \mathrm{GeV}$. The SUSY spectrum associated with these regions of parameters space could be striking signature for nonthermal scenario at the LHC.

\section{Conclusion}

We have studied the constraints imposed on the MSSM parameter space by the Higgs mass limit and the gluino lower bound, which are the most stringent collider constraints obtained from the LHC run-I at energy $8 \mathrm{TeV}$. We showed that $m_{1 / 2}$ resides within the mass range $620 \mathrm{GeV} \lesssim m_{1 / 2} \lesssim$ $2000 \mathrm{GeV}$, while the other parameters $\left(m_{0}, A_{0}, \tan \beta\right)$ are much less constrained. We also studied the effect of the measured DM relic density on the MSSM allowed parameter space. It turns out that most of the MSSM parameter space is ruled out except for few points around $\tan \beta \sim 50$, $m_{0} \sim 1 \mathrm{TeV}$, and $m_{1 / 2} \sim 1.5 \mathrm{TeV}$. We calculated the spinindependent scattering cross section of the LSP with a proton in this allowed region. We showed that our prediction for $\sigma_{\mathrm{SI}}^{p}$ is less than the recent LUX bound by at least two orders of magnitude. We have also analyzed the nonthermal DM scenario for the LSP. We showed that the constraints imposed on the MSSM parameter space are relaxed and low $\tan \beta$ is now allowed with $m_{0} \simeq \mathcal{O}(4) \mathrm{TeV}$ and $m_{1 / 2} \simeq 600 \mathrm{GeV}$. Also two allowed regions are now associated with large $\tan \beta(\sim$ 50); namely, $m_{0} \sim m_{1 / 2} \simeq \mathcal{O}(1) \mathrm{TeV}$ or $m_{0} \simeq \mathcal{O}(4) \mathrm{TeV}$ and $m_{1 / 2} \simeq 700 \mathrm{GeV}$.

\section{Conflict of Interests}

The authors declare that there is no conflict of interests regarding the publication of this paper.

\section{Acknowledgments}

This work was partially supported by the STDF Project 13858, the ICTP Grant AC-80, and the European Union FP7 ITN INVISIBLES (Marie Curie Actions, PITN-GA-2011-289442).

\section{References}

[1] P. A. R. Ade, N. Aghanim, C. Armitage-Caplan et al., "Planck 2013 results. XVI. Cosmological parameters," Astronomy \& Astrophysics, vol. 571, article A16, 2014.

[2] G. Jungman, M. Kamionkowski, and K. Griest, "Supersymmetric dark matter," Physics Report, vol. 267, no. 5-6, pp. 195-373, 1996.

[3] G. Bertone, D. Hooper, and J. Silk, "Particle dark matter: evidence, candidates and constraints," Physics Reports, vol. 405, no. 5-6, pp. 279-390, 2005.

[4] H. P. Nilles, "Supersymmetry, supergravity and particle physics," Physics Reports, vol. 110, no. 1-2, pp. 1-162, 1984.

[5] J. D. Lykken, "Introduction tosupersymmetry," In press, http:// arxiv.org/abs/hep-th/9612114.

[6] J. Wess and J. Bagger, Supersymmetry and Supergravity, Princeton University Press, Princeton, NJ, USA, 2nd edition, 1991.
[7] H. E. Haber and G. Kane, "The search for supersymmetry: probing physics beyond the standard model," Physics Reports, vol. 117, no. 2-4, pp. 75-263, 1985.

[8] S. P. Martin, "A Supersymmetry Primer," Advanced Series on Directions in High Energy Physics, vol. 21, pp. 1-153, 2010, Advanced Series on Directions in High Energy Physics, vol. 18, pp. 1-98, 1998.

[9] D. J. H. Chung, L. L. Everett, G. L. Kane, S. F. King, J. D. Lykken, and L. T. Wang, "The soft supersymmetry-breaking Lagrangian: theory and applications," Physics Reports, vol. 407, no. 1-3, pp. 1203, 2005.

[10] M. Drees, P. Roy, and R. M. Godbole, Theory and Phenomenology of Sparticles, World Scientific, Singapore, 2005.

[11] H. Baer and X. Tata, Weak Scale Supersymmetry: From Superfields to Scattering Events, Cambridge University Press, Cambridge, UK, 2006.

[12] H. Goldberg, "Constraint on the photino mass from cosmology," Physical Review Letters, vol. 50, no. 19, pp. 1419-1422, 1983, Erratum-ibid: Physical Review Letters, vol. 103, Article ID 099905, 2009.

[13] G. Aad, T. Abajyan, B. Abbott et al., "Observation of a new particle in the search for the Standard Model Higgs boson with the ATLAS detector at the LHC," Physics Letters B, vol. 716, no. 1, pp. 1-29, 2012.

[14] S. Chatrchyan, V. Khachatryan, A. M. Sirunyan et al., "Observation of a new boson at a mass of $125 \mathrm{GeV}$ with the CMS experiment at the LHC," Physics Letters B, vol. 716, no. 1, pp. 30-61, 2012.

[15] G. Aad, B. Abbott, J. Abdallah et al., "Summary of the ATLAS experiment's sensitivity to supersymmetry after LHC Run 1interpreted in the phenomenological MSSM," Journal of High Energy Physics, vol. 2015, article 134, 2015.

[16] A. Gaz, "SUSY searches at CMS," In press, http://arxiv.org/abs/ 1411.1886.

[17] I. Melzer-Pellmann and P. Pralavorio, "Lessons for SUSY from the LHC after the first run," The European Physical Journal C, vol. 74, article 2801, 2014.

[18] N. Craig, "The state ofsupersymmetry after Run I of the LHC," http://arxiv.org/abs/1309.0528.

[19] D. S. Akerib, H. M. Araújo, X. Bai et al., "First results from the LUX dark matter experiment at the Sanford Underground Research Facility," Physical Review Letters, vol. 112, Article ID 091303, 2014.

[20] E. Aprile, “The Xenon1T dark matter search experiment," in Sources and Detection of Dark Matter and Dark Energy in the Universe, vol. 148 of Springer Proceedings in Physics, pp. 93-96, Springer, 2013.

[21] E. Aprile, M. Alfonsi, K. Arisaka et al., "Dark matter results from 225 live days of XENON100 data," Physical Review Letters, vol. 109, no. 18, Article ID 181301, 6 pages, 2012.

[22] H. Baer, V. Barger, and A. Mustafayev, "Implications of a $125 \mathrm{GeV}$ Higgs scalar for the LHC supersymmetry and neutralino dark matter searches," Physical Review D, vol. 85, Article ID 075010, 2012.

[23] J. Ellis and K. A. Olive, "Revisiting the higgs mass and dark matter in the CMSSM," The European Physical Journal C, vol. 72, article 2005, 2012.

[24] O. Buchmueller, R. Cavanaugh, M. Citron et al., "The CMSSM and NUHM1 in light of $7 \mathrm{TeV}$ LHC, $B_{s} \rightarrow \mu^{+} \mu^{-}$and XENON100 data," The European Physical Journal C, vol. 72, no. 11, article 2243, 2012. 
[25] O. Buchmueller, R. Cavanaugh, A. De Roeck et al. et al., "The CMSSM and NUHM1 after LHC run 1," The European Physical Journal C, vol. 74, article 2922, 2014.

[26] R. Aaij, B. Adeva, M. Adinolfi et al., "Measurement of the $B_{s}^{0} \rightarrow$ $\mu^{+} \mu^{-}$branching fraction and search for $B^{0} \rightarrow \mu^{+} \mu^{-}$decays at the LHCb experiment," Physical Review Letters, vol. 111, no. 10, Article ID 101805, 2013.

[27] S. Chatrchyan, B. Betev, L. Caminada et al., "Measurement of the $B_{s}^{0} \rightarrow \mu^{+} \mu^{-}$branching fraction and search for $B^{0} \rightarrow \mu^{+} \mu^{-}$ with the CMS experiment," Physical Review Letters, vol. 111, no. 10, Article ID 101804, 2013.

[28] CMS and LHCb Collaborations, "Combination of results on the rare decays $B_{(s)}^{0} \rightarrow \mu^{+} \mu^{-}$from the CMS and $\mathrm{LHCb}$ experiments," Tech. Rep. CMS-PAS-BPH-13-007, CERN, 2013.

[29] D. Feldman, Z. Liu, and P. Nath, "Low mass neutralino dark matter in the minimal supersymmetric standard model with constraints from $B_{s} \rightarrow \mu^{+} \mu^{-}$and Higgs boson search limits," Physical Review D, vol. 81, no. 11, Article ID 117701, 4 pages, 2010.

[30] S. Akula, D. Feldman, P. Nath, and G. Peim, "Excess observed in CDF $B_{s}^{0} \rightarrow \mu^{+} \mu^{-}$and supersymmetry at the LHC," Physical Review D, vol. 84, no. 11, Article ID 115011, 10 pages, 2011.

[31] Y. Amhis, Sw. Banerjee, R. Bernhard et al., "Averages of bhadron, c-hadron,and tau-lepton properties as of early 2012," http://arxiv.org/abs/1207.1158.

[32] B. Bhattacherjee, M. Chakraborti, A. Chakraborty, U. Chattopadhyay, D. Das, and D. K. Ghosh, "Implications of the 98 $\mathrm{GeV}$ and $125 \mathrm{GeV}$ higgs scenarios in nondecoupling supersymmetry with updated ATLAS, CMS, and PLANCK data," Physical Review D, vol. 88, no. 3, Article ID 035011, 2013.

[33] U. Haisch and F. Mahmoudi, "MSSM: cornered and correlated," Journal of High Energy Physics, vol. 2013, no. 1, article 61, 2013.

[34] N. Chen, D. Feldman, Z. Liu, and P. Nath, "SUSY and higgs signatures implied by cancellations in $b \rightarrow s \gamma$," Physics Letters $B$, vol. 685, no. 2-3, pp. 174-181, 2010.

[35] M. E. Gómez, T. Ibrahim, P. Nath, and S. Skadhauge, "An improved analysis of $b \rightarrow s \gamma$ in supersymmetry," Physical Review D, vol. 74, no. 1, Article ID 015015, 19 pages, 2006.

[36] U. Chattopadhyay and P. Nath, " $b-\tau$ unification, $g_{\mu}-2$, the $\vec{b} s+\gamma$ constraint, and nonuniversalities," Physical Review D, vol. 65, no. 7, Article ID 075009, 2002.

[37] A. Djouadi, U. Ellwanger, and A. M. Teixeira, "Constrained next-to-minimal supersymmetric standard model," Physical Review Letters, vol. 101, no. 10, Article ID 101802, 4 pages, 2008.

[38] A. Djouadi, U. Ellwanger, and A. M. Teixeira, "Phenomenology of the constrained NMSSM," Journal of High Energy Physics, vol. 2009, no. 4, article 31, 2009.

[39] S. Khalil and A. Masiero, "Radiative B-L symmetry breaking in supersymmetric models," Physics Letters B, vol. 665, no. 5, pp. 374-377, 2008.

[40] P. Fileviez Pérez and S. Spinner, "Fate of R parity," Physical Review D, vol. 83, no. 3, Article ID 035004, 7 pages, 2011.

[41] A. Elsayed, S. Khalil, and S. Moretti, "Higgs mass corrections in the SUSY B - L model with inverse seesaw," Physics Letters B, vol. 715, no. 1-3, pp. 208-213, 2012.

[42] D. Cerdeno, C. Hugonie, D. Lopez-Fogliani, C. Munoz, and A. Teixeira, "Theoretical predictions for the direct detection of neutralino dark matter in the NMSSM," Journal of High Energy Physics, vol. 2004, no. 12, article 048, 2004.

[43] A. Menon, D. E. Morrissey, and C. E. M. Wagner, "Electroweak baryogenesis and dark matter in a minimal extension of the MSSM," Physical Review D, vol. 70, Article ID 035005, 2004.
[44] S. Khalil, H. Okada, and T. Toma, "Right-handed sneutrino dark matter in supersymmetric $B-L$ model," Journal of High Energy Physics, vol. 2011, article 26, 2011.

[45] S. Khalil and H. Okada, "Dark matter in B - L extended MSSM models," Physical Review D, vol. 79, no. 8, Article ID 083510, 9 pages, 2009.

[46] F. Staub, "SARAH 3.2: dirac gauginos, UFO output, and more," Computer Physics Communications, vol. 184, no. 7, pp. 17921809, 2013.

[47] W. Porod, "SPheno, a program for calculating supersymmetric spectra, SUSY particle decays and SUSY particle production at $e^{+} e^{-}$colliders," Computer Physics Communications, vol. 153, no. 2, pp. 275-315, 2003.

[48] W. Porod and F. Staub, "SPheno 3.1: extensions including flavour, CP-phases and models beyond the MSSM," Computer Physics Communications, vol. 183, no. 11, pp. 2458-2469, 2012.

[49] J. R. Ellis, G. Ridolfi, and F. Zwirner, "Radiative corrections to the masses of supersymmetric Higgs bosons," Physics Letters B, vol. 257, no. 1-2, pp. 83-91, 1991.

[50] J. R. Ellis, G. Ridolfi, and F. Zwirner, “On radiative corrections to supersymmetric Higgs boson masses and their implications for LEP searches," Physics Letters B, vol. 262, no. 4, pp. 477-484, 1991.

[51] H. E. Haber and R. Hemping, "Can the mass of the lightest Higgs boson of the minimal supersymmetric model be larger than $m_{z}$ ?" Physical Review Letters, vol. 66, no. 14, pp. 1815-1818, 1991.

[52] Y. Okada, M. Yamaguchi, and T. Yanagida, "Renormalizationgroup analysis on the Higgs mass in the softly-broken supersymmetric standard model," Physics Letters B, vol. 262, no. 1, pp. 54-58, 1991.

[53] S. Heinemeyer, W. Hollik, and G. Weiglein, "The mass of the lightest MSSM Higgs boson: a compact analytical expression at the two-loop level," Physics Letters B, vol. 455, no. 1-4, pp. 179191, 1999.

[54] M. Carena, H. E. Haber, S. Heinemeyer, W. Hollik, C. E. M. Wagner, and G. Weiglein, "Reconciling the two-loop diagrammatic and effective field theory computations of the mass of the lightest $\mathscr{C} \mathscr{P}$-even Higgs boson in the MSSM," Nuclear Physics $B$, vol. 580, no. 1-2, pp. 29-57, 2000.

[55] J. R. Espinosa and R. J. Zhang, "Complete two-loop dominant corrections to the mass of the lightest $\mathscr{C} \mathscr{P}$-even Higgs boson in the minimal supersymmetric standard model," Nuclear Physics $B$, vol. 586, no. 1-2, pp. 3-38, 2000.

[56] G. Aad, B. Abbott, J. Abdallah et al., "Search for squarks and gluinos in events with isolated leptons, jets and missing transverse momentum at $\sqrt{s}=8 \mathrm{TeV}$ with the ATLAS detector," Journal of High Energy Physics, vol. 2015, no. 4, article 116, 2015.

[57] S. Chatrchyan, V. Khachatryan, A. M. Sirunyan et al., "Search for gluino mediated bottom- and top-squark production in multijet final states in pp collisions at $8 \mathrm{TeV}$," Physics Letters B, vol. 725, no. 4-5, pp. 243-270, 2013.

[58] H. E. Haber and G. L. Kane, "The search for supersymmetry: probing physics beyond the standard model," Physics Reports, vol. 117, no. 2-4, pp. 75-263, 1985.

[59] J. F. Gunion and H. E. Haber, "Higgs bosons in supersymmetric models," Nuclear Physics B, vol. 272, no. 1, pp. 1-76, 1986, Erratum: Nuclear Physics B, vol. 402, pp. 567-569, 1993.

[60] M. M. El Kheishen, A. A. Shafik, and A. A. Aboshousha, "Analytic formulas for the neutralino masses and the neutralino mixing matrix," Physical Review D, vol. 45, article 4345, 1992. 
[61] M. Guchait, "Exact solution of the neutralino mass matrix," Zeitschrift für Physik C, vol. 57, no. 1, pp. 157-163, 1993, Erratum in: Zeitschrift für Physik C, vol. 61, p. 178, 1994.

[62] E. W. Kolb and M. S. Turner, The Early Universe, vol. 70 of Frontier in Physics, Addison-Wesley, Redwood City, Calif, USA, 1988.

[63] G. Belanger, F. Boudjema, A. Pukhov, and A. Semenov, "micrOMEGAs_3: a program for calculating dark matter observables," Computer Physics Communications, vol. 185, no. 3, pp. 960-985, 2014.

[64] M. Chakraborti, U. Chattopadhyay, S. Rao, and D. P. Roy, "Higgsino dark matter in nonuniversal gaugino mass models," Physical Review D, vol. 91, no. 3, Article ID 035022, 2015.

[65] K. Kowalska, L. Roszkowski, and E. M. Sessolo, "Two ultimate tests of constrained supersymmetry," Journal of High Energy Physics, vol. 2013, article 78, 2013.

[66] G. F. Giudice, E. W. Kolb, and A. Riotto, "Largest temperature of the radiation era and its cosmological implications," Physical Review D, vol. 64, no. 2, Article ID 023508, 2001.

[67] T. Moroi and L. Randall, "Wino cold dark matter from anomaly mediated SUSY breaking," Nuclear Physics B, vol. 570, no. 1-2, pp. $455-472,2000$. 

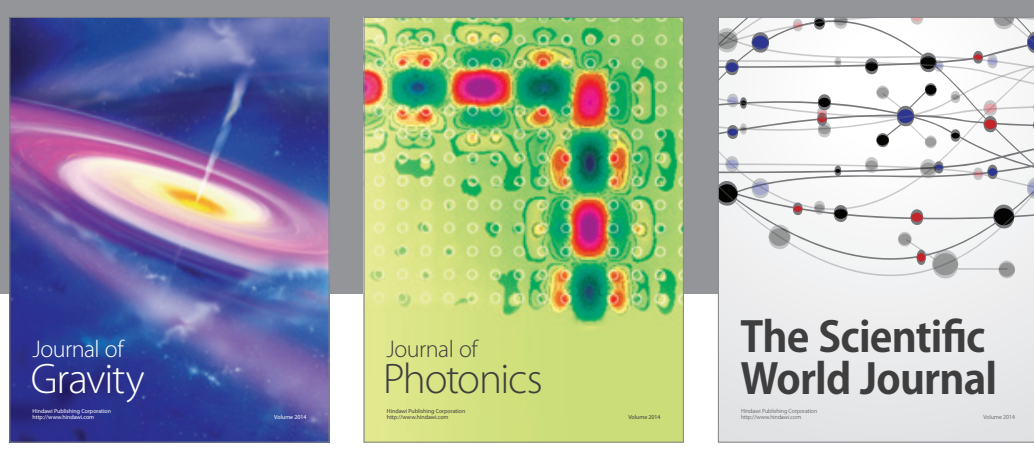

The Scientific World Journal
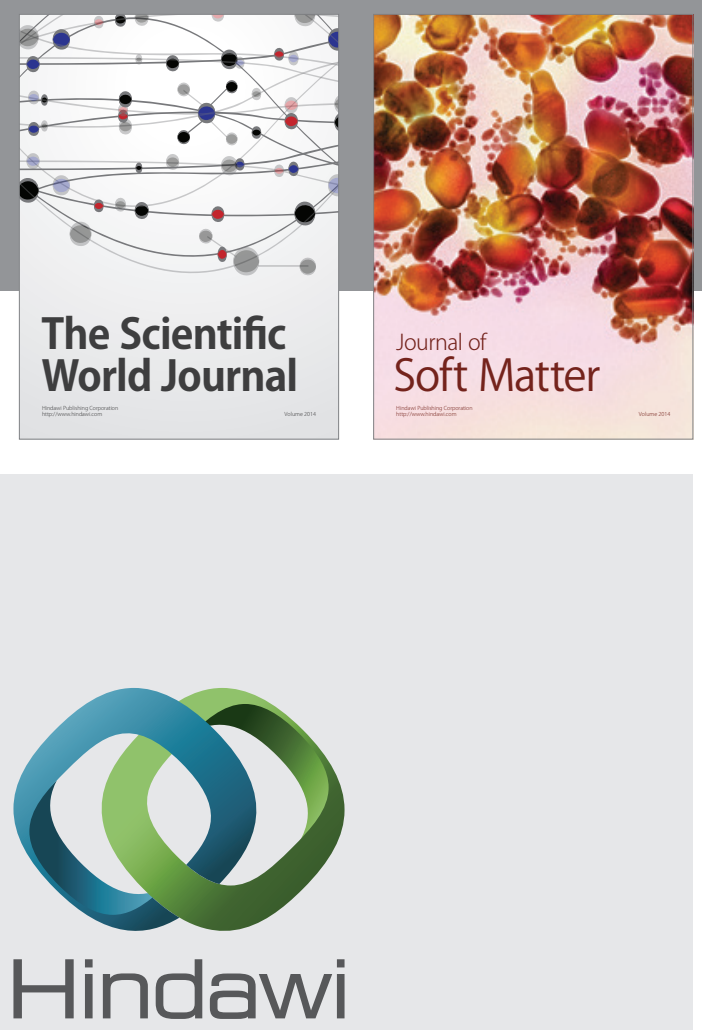

Submit your manuscripts at

http://www.hindawi.com

nternational Journal of

Statistical Mechanics
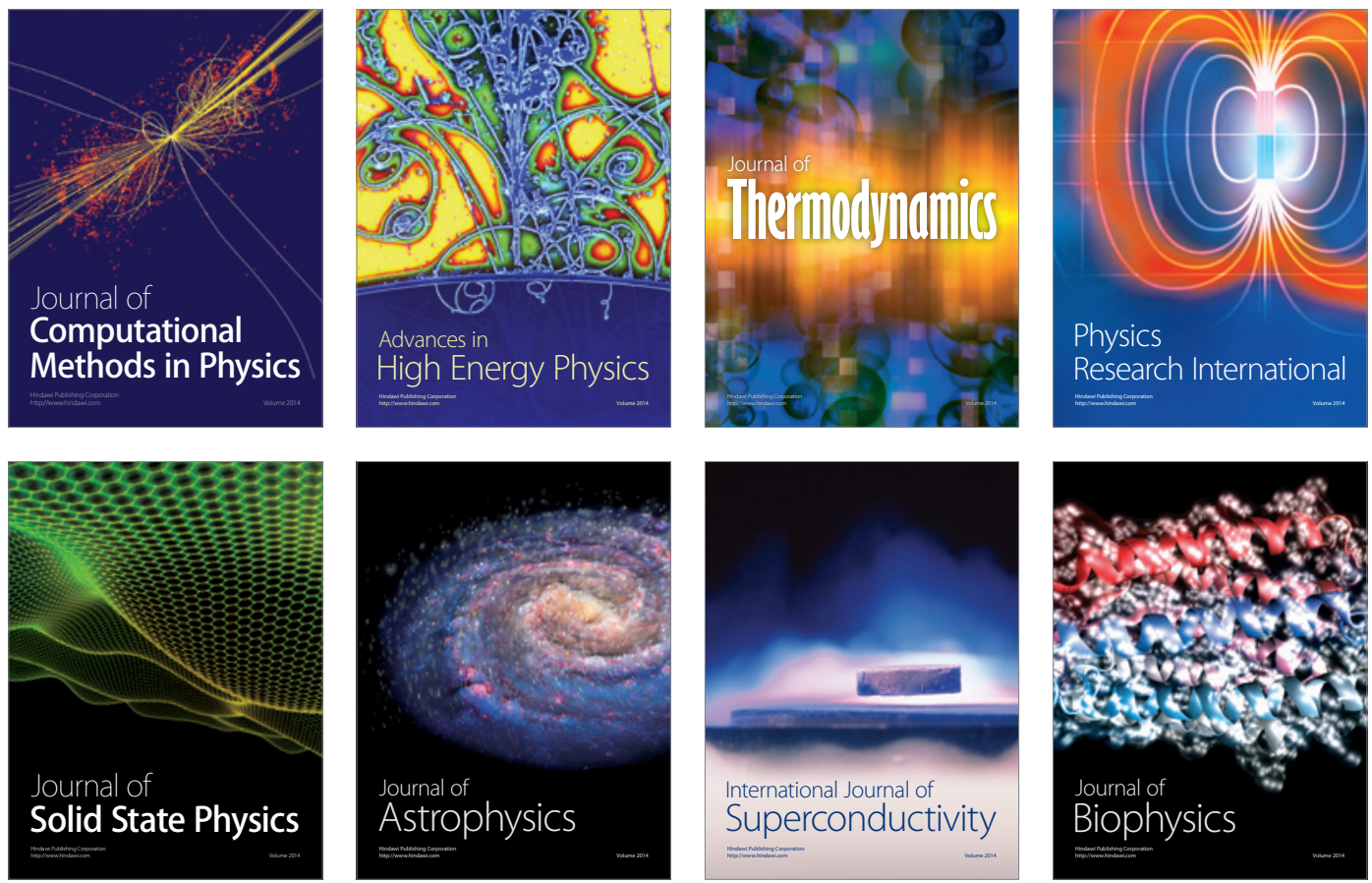
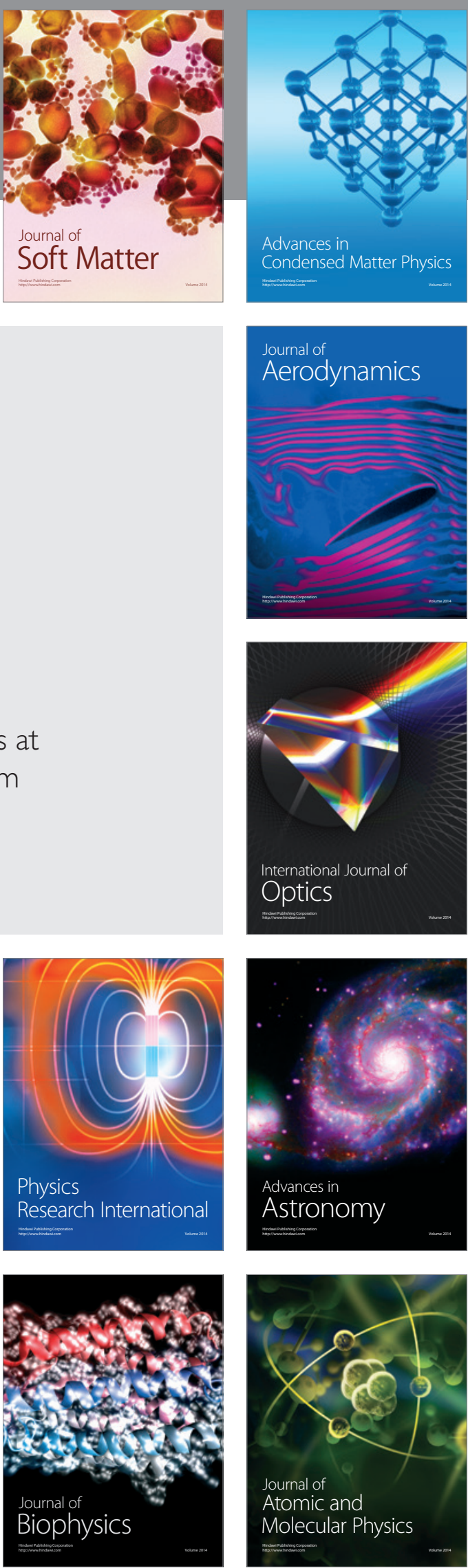